\title{
Boxcar detection for high-frequency modulation in stimulated Raman scattering microscopy
}

\author{
P. Fimpel, ${ }^{1}$ C. Riek, ${ }^{2}$ L. Ebner, ${ }^{1}$ A. Leitenstorfer, ${ }^{2}$ D. Brida,${ }^{2}$ and A. Zumbusch ${ }^{1}$ \\ ${ }^{1}$ Department of Chemistry and Center for Applied Photonics (CAP), University of Konstanz, \\ D-78457 Konstanz, Germany \\ ${ }^{2}$ Department of Physics and Center for Applied Photonics (CAP), University of Konstanz, D-78457 Konstanz, \\ Germany
}

\begin{abstract}
Stimulated Raman scattering (SRS) microscopy is an important non-linear optical technique for the investigation of unlabeled samples. The SRS signal manifests itself as a small intensity exchange between the laser pulses involved in coherent excitation of Raman modes. Usually, high-frequency modulation is applied in one pulse train, and the signal is then detected on the other pulse train via lock-in amplification. While allowing shot-noise limited detection sensitivity, lock-in detection, which corresponds to filtering the signal in the frequency domain, is not the most efficient way of using the excitation light. In this manuscript, we show that boxcar averaging, which is equivalent to temporal filtering, is better suited for the detection of low-duty-cycle signals as encountered in SRS microscopy. We demonstrate that by employing suitable gating windows, the signal-to-noise ratios achievable with lock-in detection can be realized in shorter time with boxcar averaging. Therefore, high-quality images are recorded at a faster rate and lower irradiance which is an important factor, e.g., for minimizing degradation of biological samples.
\end{abstract}

Non-linear optical Raman microscopy has become a valuable diagnostics tool in the biological, medical, and materials sciences. ${ }^{1-4}$ Two different approaches are currently employed, namely Coherent Anti-Stokes Raman Scattering (CARS) microscopy ${ }^{5,6}$ and stimulated Raman scattering (SRS) microscopy. ${ }^{7-9}$ With respect to sensitivity, ${ }^{10}$ both methods cannot compete with fluorescence techniques. In contrast to the latter, however, they offer the possibility to obtain contrast in unlabelled samples. In both Raman techniques, molecular specificity is achieved by tuning the frequency difference between the exciting pump and Stokes lasers to match the energy of a vibrational excitation in the sample. Lately, SRS microscopy has received a lot of attention since it does not display a non-resonant electronic background ${ }^{11}$ and its signal depends linearly on the molecular concentration. Thus, quantitative data analysis is more reliable with SRS as compared to CARS microscopy. Concerning the detection scheme, the two methods are distinctly different. In CARS microscopy, the signal is shifted to wavelengths shorter than those of the excitation light and therefore easily acquired after simple optical filtering. SRS microscopy, by contrast, requires the detection of the signal at the frequency of one of the excitation lasers, in the case of stimulated Raman loss (SRL) detection at the pump wavelength, for stimulated Raman gain (SRG) detection at the Stokes wavelength. Only a minute fraction of the excitation light is Raman scattered, so that the SRS signal appears as small modulation on top of an intense background. For its detection, sensitivities on the order of $10^{-6}$ or better are typically required. Most SRS investigations have relied on lock-in detection of the SRS signal with high-frequency modulation. This approach allows shot-noise-limited measurements at the cost of imposing a strong filter function at the demodulation frequency of the lock-in. Recently, also different electronic filters relying on specially designed detectors were employed for SRS microscopy. ${ }^{12,13}$ A general problem for SRS microscopy consists in the fact that in order to reach the targeted sensitivity range, a sufficiently large number of photons must be collected. This goal is usually achieved by increasing the excitation intensity. However, this strategy is at odds with the prerequisites for studying biological samples which commonly do not withstand high excitation powers. Therefore, an optimization of the detection process is vital for implementing the most efficient use of the excitation photons. In addition, SRS microscopy is performed with pulses of picosecond durations at repetition rates of several tens of $\mathrm{MHz}$ in order to effectively drive the non-linear SRS process while preserving spectral selectivity in the excitation of Raman vibrational modes. These experimental conditions imply low-duty-cycle signals, and lock-in detection is not the best approach for signal recovery. Here, we demonstrate that boxcar averaging is superior to lock-in detection in experiments of this type.

The general approach to boxcar averaging is based on time-domain integration of a periodic signal in a specific temporal window that is defined as a gate. Its duration is optimized to overlap the time-domain response of the detectors only where information is contained. Like this, electronic noise contributions outside the gating window are suppressed. In the case of a periodic train of pulses, each of these is sampled individually and then averaged with a constant weight. By contrast, lock-in detection operates in the frequency domain by acting as a narrow filter at the modulation frequency of the signal. This signal recovery scheme is extremely efficient for harmonic modulation, in particular at high frequencies where fast analog gating electronics has traditionally not been available. However, the lock-in approach is suboptimal in experiments that employ a coherent pulse 
train produced by mode-locked lasers where filtering a narrow frequency band at the modulation frequency does not match the low duty cycle of the periodic signal detected. In addition, lock-in amplifiers use a moving average with an exponential weight of the signal that hampers the requirement of fast optical experiments. For these reasons, boxcar averaging has historically been the method of choice for the analysis of periodic signals with low repetition rates, while applications at high repetition rates $(\mathrm{MHz}$ and higher) were preferentially processed with lock-in detection. This situation has changed recently with the advent of fast digital boxcar electronics suited for the analysis of high-frequency de-modulation of signals as encountered, e.g., in imaging applications driven by laser systems that operate at multi$\mathrm{MHz}$ repetition rates. In the following, we describe how boxcar averaging can be used in SRS microscopy, show that it is superior to lock-in detection in this application with respect to sensitivity, and discuss the details of its experimental implementation.

Our experimental setup is based on a two-arm Er:fiber laser system which is sketched in Fig. 1 and has been introduced in detail earlier. ${ }^{14,15}$ In brief, an Er:fiber oscillator (Er:OSC) seeds two Er:fiber amplifiers (EDFAs). In the first arm, the amplifier operates at a repetition rate of $40 \mathrm{MHz}$, and its output is frequency-doubled in a periodically poled $\mathrm{LiNbO}_{3}$ (PPLN) crystal, resulting in a train of pulses with an average power of $75 \mathrm{~mW}$ and a central wavelength tunable between $765 \mathrm{~nm}$ and $781 \mathrm{~nm}$. These pulses serve as pump pulses in coherent Raman excitation experiments. In the second arm, the amplifier is seeded after picking out every second pulse from the oscillator with an electro-optic modulator (EOM). In this way, the repetition rate is reduced to the Nyquist frequency of $20 \mathrm{MHz}$. The pulse train of this amplifier is coupled into a highly non-linear optical fiber to generate a broadband soliton which is subsequently frequency doubled in a fan-out PPLN. The output beam has an average power of up to $10.5 \mathrm{~mW}$. It is tunable between $809 \mathrm{~nm}$ and $1030 \mathrm{~nm}$. Since they originate from the same oscillator, pump and Stokes pulses are perfectly synchronized without any significant timing jitter. ${ }^{16}$ The pulse durations are approximately $2 \mathrm{ps}$, resulting in a duty cycle of the all-optical signal of $4 \times 10^{-5}$. Due to the finite detection bandwidth of photodetectors, the electrical duty cycle is larger than the optical duty

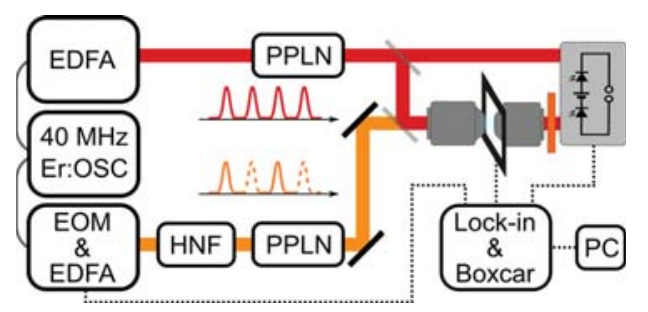

FIG. 1. Schematic layout of the experimental setup. The Er:fiber oscillator (Er:OSC) synchronously pumps two Er:fiber amplifiers (EDFA). In the first arm, the EDFA output is frequency doubled in a PPLN. This branch is the pump beam in the SRS microscopy experiment. In the second arm, the Er:OSC output is pulse picked at $20 \mathrm{MHz}$ with a fiber coupled electro-optic modulator (EOM). This output is coupled into a highly nonlinear fiber (HNF), and the generated soliton is frequency doubled in a fan-out PPLN producing the Stokes beam. The output of a balanced detector is processed with either a lock-in amplifier or a boxcar averager. cycle. The tunability of both excitation beams allows the access of vibrational resonances between $443 \mathrm{~cm}^{-1}$ and $3363 \mathrm{~cm}^{-1}$.

For the measurements reported here, the SRS signal was acquired with a balanced photodetector (PDB450A, Thorlabs, USA) which was set to a bandwidth of $150 \mathrm{MHz}$ and an amplification factor of 500. The stimulated Raman loss (SRL) of the pump laser was detected as a signal after rejection of the Stokes wavelength by a combination of a shortpass (FESH0800, Thorlabs, USA) and a bandpass filter (Z780/30 $\mathrm{X}$, Chroma, USA). The output of the detector was fed into the input of a digital measurement device, providing a lock-in amplifier and boxcar averaging capabilities (UHFLI, Zürich Instruments, Switzerland). In order to compare lock-in detection and boxcar averaging, two configurations were employed in which either the native output of the balanced photodetector or the same output filtered with an electronic bandpass filter centered at $21 \mathrm{MHz}$ with a width of $2 \mathrm{MHz}$ (BBP-21.4+, Mini-Circuits, USA) was used. Balanced detection was applied in order to eliminate the amplitude noise of the Er:fiber laser such that shot-noise-limited signal detection is possible. ${ }^{15,17,18}$ This strategy also has the advantage of reducing the amplitude of the electronic signal passed onto the high-frequency lock-in/boxcar in order to maximize the signal resolution at the analog-to-digital conversion.

The difference in the operation of the lock-in amplifier and the boxcar averager was tested by recording the SRL signal from a droplet of benzonitrile over $10 \mathrm{~s}$ simultaneously with both methods. The optical focus was kept at a fixed position in the sample. A $25 \times 0.95 \mathrm{NA}$ water objective and a 1.4 NA oil condenser (both Leica microsystems, Germany) were used. Figure 2 depicts the operation regime of the electronic signals fed to the boxcar/lock-in for the case in which the native output of the balanced detector was exploited. Figure 2(a) shows the electronic output of the detector that measures the pulse train of the Raman pump at $40 \mathrm{MHz}$ modulated by the nonlinear interaction with the $20 \mathrm{MHz}$ Stokes beam incident on the sample. The waveform shows one complete period $\left(360^{\circ}\right)$ at the demodulation frequency, which corresponds to $50 \mathrm{~ns}$ indicated on the respective horizontal axis. This electronic waveform was employed for the detection of the SRS signal. As described above for boxcar averaging, the acquisition occurs by averaging the voltage readout over the gating time. Under our experimental conditions, this temporal window was set to $7.2 \mathrm{~ns}$ (area shaded in gray in Fig. 2). The boxcar averager unit calculates the difference between the distinct mean signal value of the area (W1) and (W2), which yields the voltage at the boxcar output. This boxcar voltage is proportional to the differential SRS signal. It is internally averaged over an arbitrary number of pulse pairs. This operation is conceptually equivalent to calculating the area underlying the difference between the waveform of the Raman pump pulse with and without the Stokes excitation depicted in Fig. 2(b) and to subsequent division of this value by the temporal duration of the gating window. In the case of lock-in detection, the time constant was set to $5 \mathrm{~ms}$ using a 4 th order filter. Therefore, every $50 \mathrm{~ms}$, an independent measurement point was acquired, yielding 200 points over $10 \mathrm{~s}$ for the determination of the signal-to-noise ratio (SNR) achievable with this demodulation approach. The 

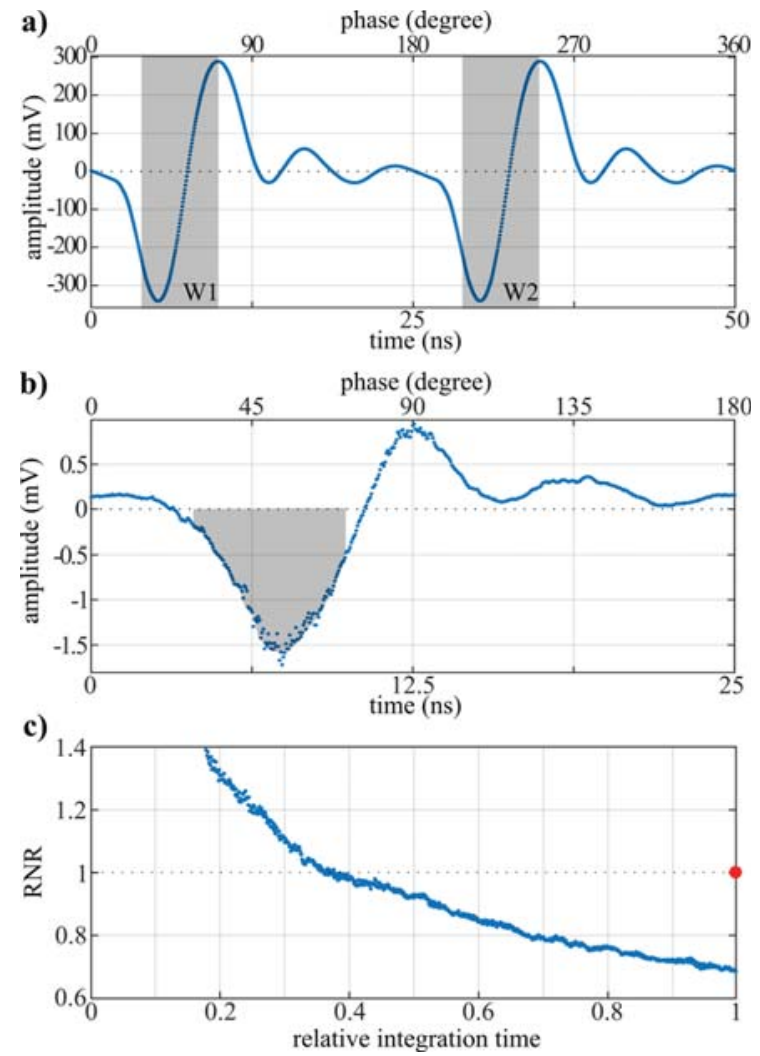

FIG. 2. (a) Waveform of the voltage signal of the balanced detector with the shaded areas indicating the gating windows employed for boxcar detection (W1 and W2). The lower axis represents time in one demodulation period, whereas the top axis corresponds to the phase in one demodulation period. Two Raman pump pulses, one with simultaneous Stokes excitation and one without, are visible. The difference between the signals of these two pulses is plotted in (b) to visualize the stimulated Raman loss due to the Stokes pulse incident during W1. The value detected by the boxcar is proportional to the shaded area under this curve. (c) Relative noise ratio (RNR) between the noise level achieved with boxcar averaging and the one obtained with lock-in detection (red dot at relative integration time $=1$ ) as a function of the integration time of the boxcar averager relative to 10 time constants of the lock-in amplifier.

waiting time of 10 time constants is required to ensure that the values of two adjacent values are uncorrelated. By contrast, the boxcar averager was set to a moving average over 1024 reference periods with an equal weight. Consequently, a readout was recorded every $52 \mu \mathrm{s}$, and approximately 1000 data points were registered in the same temporal duration of $50 \mathrm{~ms}$. Figure 2(c) shows the relative noise ratio (RNR) in the dependence of the integration time $\left(t_{\text {int }}\right)$

$$
\operatorname{RNR}\left(\mathrm{t}_{\text {int }}\right)=\frac{\mathrm{SNR}_{\text {Lock-in }}}{\operatorname{SNR}_{\text {Boxcar }}\left(\mathrm{t}_{\text {int }}\right)} .
$$

The SNR for each filtering technique is the mean signal over a $10 \mathrm{~s}$ measurement divided by the standard deviation of the data points every $50 \mathrm{~ms}$. The measurement time for the lockin detection is fixed to $50 \mathrm{~ms}$ due to the applied time constant, whereas the boxcar integration time is varied from 52 $\mu \mathrm{s}$ to $50 \mathrm{~ms}$ by taking $1-1000$ data points, and the corresponding SNR is evaluated as a function of the averaging time as depicted in Fig. 2(c). By comparing the noise amplitudes for both filter techniques, one can evaluate an SNR that is 1.4 times better than achievable with lock-in detection when the same dwell time is considered. Remarkably, the boxcar averager achieves the same SNR within a 0.4 fraction of the time the lock-in amplifier requires. This corresponds to a reduction of the sample irradiance by a factor of 2.5 which is an important criterion for the avoidance of photodamage in investigations of biological samples. ${ }^{19}$

Figure 3 shows the same characterization with the electronic filtering of the signal at the modulation frequency of $20 \mathrm{MHz}$. Here, the high frequencies contained in the native signal that will not be detected by the lock-in amplifier are pre-filtered. The result is an almost harmonic detection voltage waveform that is ideal for lock-in detection as can be encountered in systems in which the modulation frequency is set to be lower than half the repetition rate of the laser. It should be noted that the gating window was kept at 7.2 ns and not further optimized to have a better temporal overlap with the filtered output of the balanced photodetector. Despite this, by the same analyses as from the previous configuration, the boxcar detection still produces a final SNR that beats the lock-in by a factor of 1.24. Comparable SNR performances are achieved with a dwell time that for boxcar averaging is less than half the one necessary for lock-in amplification. The fact that in the case with no electronic filters the same noise level $(\mathrm{RNR}=1)$ is reached earlier is explained by the higher frequency components which are detected by averaging the electronic waveforms in the time domain.

In order to demonstrate that the advantages of boxcar averaging can be directly transferred to scanning microscopy applications, we imaged polymethylmethacrylate (PMMA)
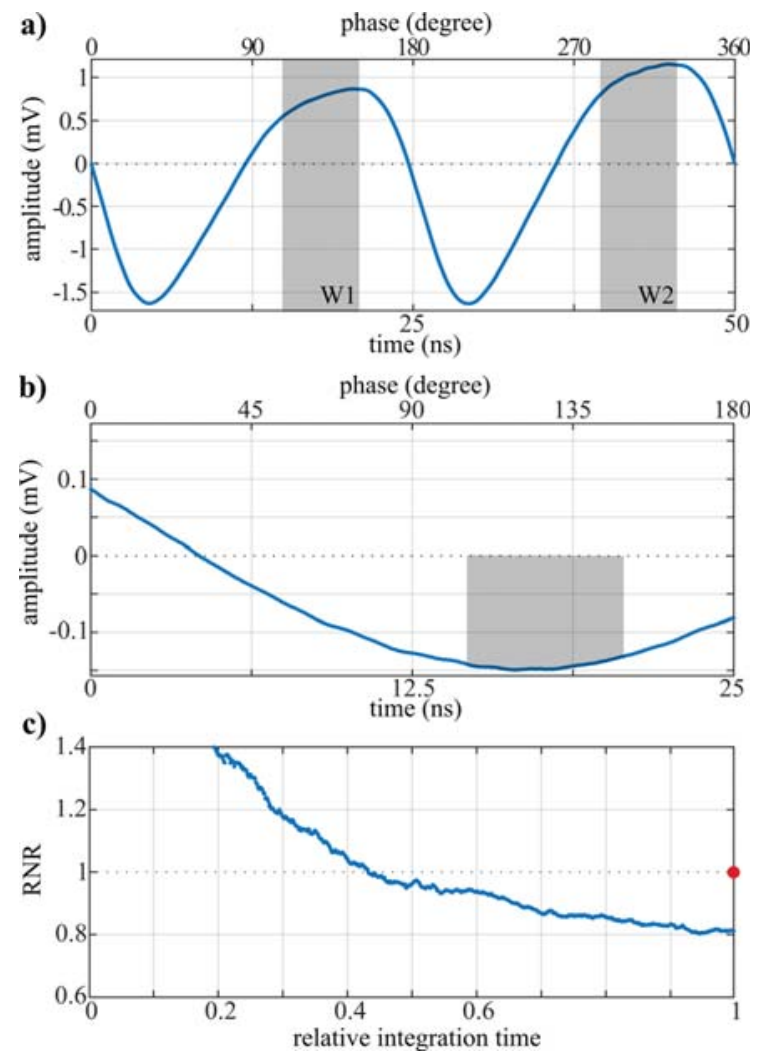

FIG. 3. (a)-(c) Voltage signals and relative noise ratios (RNRs) as described in Fig. 2 but acquired with a $21 \mathrm{MHz}$ electronic prefilter at the output of the balanced photodetector. 

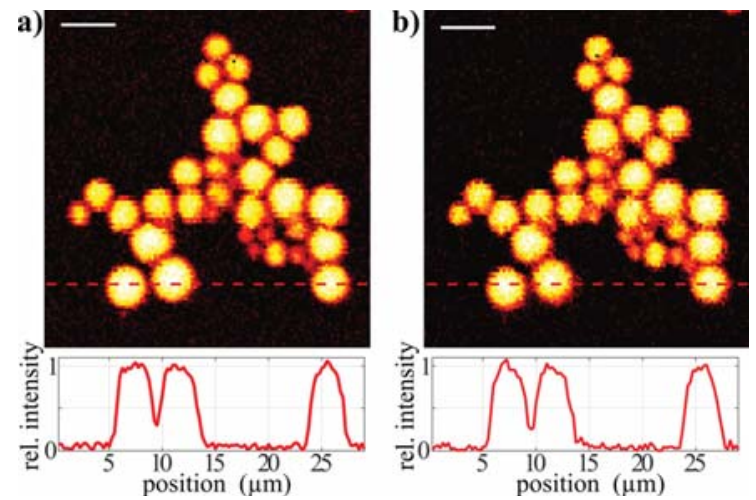

FIG. 4. Stimulated Raman loss image of a mixture of polymethylmethacrylate beads in water at a Raman shift of $2949 \mathrm{~cm}^{-1}$ recorded with lock-in detection (a) and boxcar recovery (b). The pixel dwell time was $14 \mathrm{~ms}$ (a) and $6.7 \mathrm{~ms}$ (b). The excitation power was $12 \mathrm{~mW}$ at $765.3 \mathrm{~nm}$ (pump) and $3 \mathrm{~mW}$ at $988.4 \mathrm{~nm}$ (Stokes). As a comparison for the achieved signal to noise ratio, the lower panel depicts the signal along the red dashed line in the image. The scale bar is $5 \mu \mathrm{m}$.

beads with diameters between $1 \mu \mathrm{m}$ and $5 \mu \mathrm{m}$ in water using boxcar averaging and lock-in detection (Fig. 4). The images were recorded using a $63 \times 1.2 \mathrm{NA}$ water objective and a 1.4 NA oil condenser (both Leica Microsystems, Germany). For lock-in filtering, a time constant of $1.43 \mathrm{~ms}$ using a 4th order filter was employed. This setting allows $14 \mathrm{~ms}$ pixel dwell times. For boxcar recovery, the system was configured to make a moving average over 131072 periods of the reference frequency, corresponding to a pixel dwell time of $6.7 \mathrm{~ms}$. The line plots in Fig. 4 show the signal along the dashed line in the image. The two images demonstrate that a detection scheme based on a boxcar averager reaches the same result in terms of SNR with a pixel dwell time that is half as long as that used for lock-in detection.

In conclusion, we describe boxcar averaging as a valuable technique for the detection of high-frequency modulated optical signals with low duty cycles in which the signal of two adjacent acquisition events can be widely different. For the example of SRS microscopy, a benchmark non-linear optical microscopy technique, we show that boxcar averaging is more efficient than traditional lock-in detection. This fact either allows to achieve similar SNR values for significantly shorter integration times and thus less sample irradiances or to improve data quality at the same integration times. These are important advances especially for the investigation of biological samples. While our results were obtained with a fiber laser system in combination with a balanced detection scheme, even larger gains in detection efficiency are expected for experiments based on free-space solid-state lasers, which can be employed without balancing of the readout pulses. It should also be noted that our results are not restricted to non-linear Raman microscopy but can instead be directly applied to any type of pump-probe spectroscopy and microscopy. ${ }^{20,21}$

Financial support by Deutsche Forschungsgemeinschaft, No. TP B3, SFB767 is gratefully acknowledged.

${ }^{1}$ C. Y. Chung, J. Boik, and E. O. Potma, Annu. Rev. Phys. Chem. 64, 77 (2013).

${ }^{2}$ C. H. Camp and M. T. Cicerone, Nat. Photonics 9, 295-305 (2015).

${ }^{3}$ J. X. Cheng and X. S. Xie, Science 350, aaa8870 (2015).

${ }^{4}$ I. W. Schie, C. Krafft, and J. Popp, Analyst 140, 3897 (2015).

${ }^{5}$ M. D. Duncan, J. Reintjes, and T. J. Manuccia, Opt. Lett. 7, 350 (1982).

${ }^{6}$ A. Zumbusch, G. R. Holtom, and X. S. Xie, Phys. Rev. Lett. 82, 4142 (1999).

${ }^{7}$ E. Ploetz, S. Laimgruber, S. Berner, W. Zinth, and P. Gilch, Appl. Phys. B 87, 389 (2007).

${ }^{8}$ C. W. Freudiger, W. Min, B. G. Saar, S. Lu, G. R. Holtom, C. W. He, J. C. Tsai, J. X. Kang, and X. S. Xie, Science 322, 1857 (2008).

${ }^{9}$ P. Nandakumar, A. Kovalev, and A. Volkmer, New. J. Phys. 11, 033026 (2009).

${ }^{10}$ Y. Ozeki, F. Dake, S. Kajiyama, K. Fukui, and K. Itoh, Opt. Express 17, 3651 (2009).

${ }^{11}$ P. Berto, E. R. Andresen, and H. Rigneault, Phys. Rev. Lett. 112, 053905 (2014).

${ }^{12}$ M. Slipchenko, R. A. Oglesbee, D. Zhang, W. Wu, and J.-X. Cheng, J. Biophotonics 5, 801 (2012).

${ }^{13}$ Z. Wang, W. Zheng, and Z. W. Huang, Opt. Lett. 41, 3960 (2016).

${ }^{14}$ G. Krauss, T. Hanke, A. Sell, D. Träutlein, A. Leitenstorfer, R. Selm, M. Winterhalder, and A. Zumbusch, Opt. Lett. 34, 2847 (2009).

${ }^{15}$ C. Riek, C. Kocher, P. Zirak, C. Kölbl, P. Fimpel, A. Leitenstorfer, A. Zumbusch, and D. Brida, Opt. Lett. 41, 3731 (2016).

${ }^{16}$ F. Adler, A. Sell, F. Sotier, R. Huber, and A. Leitenstorfer, Opt. Lett. 32, 3504 (2007).

${ }^{17}$ A. Gambetta, V. Kumar, G. Grancini, D. Polli, R. Ramponi, G. Cerullo, and M. Marangoni, Opt. Lett. 35, 226 (2010).

${ }^{18}$ F. Crisafi, V. Kumar, T. Scopigno, M. Marangoni, G. Cerullo, and D. Polli, Sci. Rep. 7, 10745 (2017).

${ }^{19}$ Y. Fu, H. F. Wang, R. Y. Shi, and J. X. Cheng, Opt. Express 14, 3942 (2006).

${ }^{20}$ D. Polli, G. Grancini, J. Clark, M. Celebrano, T. Virgili, G. Cerullo, and G. Lanzani, Adv. Mater. 22, 3048 (2010).

${ }^{21}$ M. C. Fischer, J. W. Wilson, F. E. Robles, and W. S. Warren, Rev. Sci. Instrum. 87, 031101 (2016). 